\title{
Performance Analysis of Time of Arrival Estimation on OFDM Signals
}

\author{
Marco Driusso*, Massimiliano Comisso, Fulvio Babich, and Chris Marshall
}

\begin{abstract}
This paper characterizes the error performance of realistically modelled orthogonal frequency division multiplexing (OFDM) signals, when their time of arrival has to be estimated in an additive white Gaussian noise channel. In particular, different power distributions on the available sub-carriers of the OFDM signal are considered, and bounds on the corresponding root mean square estimation error (RMSEE) are evaluated. The tools used for such purpose are the widely adopted Cramér-Rao bound and the Ziv-Zakai bound, which is tight in a wide range of signalto-noise ratio (SNR) values. The presented analysis reveals that, for a given signal bandwidth, a proper power distribution on the OFDM sub-carriers is crucial for achieving a good performance in the low to medium SNR region, where the RMSEE curve exhibits the typical threshold behavior. Moreover, a trade-off between asymptotic and threshold performance is identified, thanks to the adoption of a novel performance figure, which directly describes the threshold RMSEE behavior.
\end{abstract}

Index Terms-Time of arrival estimation, OFDM, root mean square, Cramér-Rao bound, Ziv-Zakai bound.

\section{INTRODUCTION}

In the recent decade, orthogonal frequency division multiplexing (OFDM) has become a successful and widely used wireless communication technique, which, thanks to its robustness and low complexity, has been adopted in a conspicuous number of wireless communication standards, including $3 \mathrm{GPP}$ LTE, DVB-T, DAB, and IEEE 802.11agn [1].

The ubiquity of this communication technique has determined an interest in the OFDM technology also in the field of positioning, where the intrinsic characteristics of the OFDM signals may be studied for understanding how to design a proper OFDM waveform suitable for pseudo-range calculation. Concerning this aspect, the estimation of the time of arrival (ToA) of a signal is a fundamental topic. Accordingly, the design of ToA-based positioning systems that exploit OFDM waveforms has been addressed in the literature [2][10], also analyzing the possibility to use band-limited multicarrier modulations for achieving selective accuracy in global navigation satellite systems [11]. Proper shaping strategies for the OFDM tones have been further adopted for joint capacity maximization and ToA accuracy purposes in multipath channels [12]-[14]. However, a careful investigation that jointly considers the asymptotic and the threshold ToA estimation performance of realistically modelled OFDM signals, and its

Marco Driusso, Massimiliano Comisso, and Fulvio Babich are with the Department of Engineering and Architecture, University of Trieste, Italy (email: marco.driusso@phd.units.it, mcomisso@units.it, babich@units.it).

Chris Marshall is with u-blox UK Ltd, Reigate, United Kingdom (email: chris.marshall@u-blox.com).

This work is partly supported by u-blox Italia SpA and by the University of Trieste within the project FRA 2013 "Multi-packet communication in 802.11x heterogeneous mobile networks: models and antenna system algorithms". dependence on the sub-carriers' power distribution, remains a partly unexplored issue.

To address this issue, which requires further deepens both for the additive white Gaussian noise (AWGN) channel and for the multipath channel, this paper characterizes the ToA estimation performance of OFDM waveforms in the AWGN case, with the purpose to provide a best-case ranging accuracy indication. The characterization is performed by properly shaping the power spectral density of the OFDM signals, so as to infer the influence of the sub-carriers' power distribution on the ToA root mean square estimation error (RMSEE), using the Cramér-Rao bound (CRB) and the Ziv-Zakai bound (ZZB) [15], [16]. Moreover, the ToA RMSEE behavior is explored not only in the asymptotic region (AR), but also in the threshold region (TR), by introducing a novel quantity, which is complementary to the widely adopted Gabor bandwidth (GB). Finally, a trade-off between asymptotic and threshold performance is identified, showing that the GB is not the only parameter to consider for designing a ToA reference signal.

The paper is organized as follows. Section II introduces the system model. Section III analyzes the ToA estimation performance. Section IV discusses the numerical results. Section V summarizes the most relevant conclusions.

\section{SySTEM MODEL}

\section{A. OFDM signal model}

The proposed investigation considers a realistic OFDM signal, consisting of $N_{\mathrm{c}}$ used sub-carriers out of $N_{\mathrm{dft}}>N_{\mathrm{c}}$ available ones. A set of $N_{\mathrm{c}} / 2$ used sub-carriers is arranged symmetrically on each side of the direct current (DC) subcarrier, which is assumed to be empty, and the reminder of the spectrum is padded out with empty sub-carriers, $\left(N_{\mathrm{dft}}-N_{\mathrm{c}}\right) / 2$ in the negative range and $\left(N_{\mathrm{dft}}-N_{\mathrm{c}}\right) / 2-1$ in the positive range. Hence, the parameter $N_{\mathrm{c}}$ controls the available bandwidth for each considered OFDM waveform. The ideal complex baseband representation of the described OFDM signal may be expressed as [1]:

$$
s(t)=\sum_{k=-N_{\mathrm{c}} / 2}^{N_{\mathrm{c}} / 2} g(t) S[k] e^{j 2 \pi k \Delta f t}, \quad t \in[0, T],
$$

where $g(t)$ is the shaping impulse, $S[k] \in \mathbb{C}$ is the symbol transmitted on the $k^{\text {th }}$ sub-carrier (with $S[0]=0$ ), $\Delta f=1 / T$ is the inter-carrier frequency separation, and $T$ is the symbol duration. The vector $\mathbf{S}=\left[S\left[-N_{\mathrm{c}} / 2\right], \ldots, S[k], \ldots, S\left[N_{\mathrm{c}} / 2\right]\right] \in$ $\mathbb{C}^{N_{c}+1}$ must satisfy the energy constraint $\|\mathbf{S}\|^{2}=1$. Ideally, $g(t)$ should be the rectangular function. However, in real world implementations, $g(t)$ is determined by the digital to analog 
converter (DAC), which generates the continuous time signal:

$$
s_{\mathrm{c}}(t)=\sum_{n=0}^{N_{\mathrm{dft}}-1} s[n] p_{w}\left(t-n T_{s}\right),
$$

where $s[n]=s\left(t=n T_{s}\right)$ is the sampled ideal OFDM signal with $T_{s}=T / N_{\mathrm{dft}}$ denoting the sampling interval, and $p_{w}(t)=$ $w(t) \cdot \operatorname{sinc}\left(t / T_{s}\right)$ is the impulse response of the DAC with $w(t)$ denoting the windowing function that limits in time the sinc interpolating function. The set of the $N_{\mathrm{dft}}$ samples $\mathbf{s}=$ $\left[s[0], \ldots, s\left[N_{\mathrm{dft}}-1\right]\right] \in \mathbb{C}^{N_{\mathrm{dft}}}$ is usually obtained through a length $N_{\text {dft }}$ inverse DFT (IDFT) operation on the sequence $\mathbf{S}$. More particularly, $\mathbf{s}=\operatorname{IDFT}_{N_{\text {dft }}}\{\tilde{\mathbf{S}}\}$, where:

$$
\tilde{\mathbf{S}}=\left[S[0], \ldots, S\left[N_{\mathrm{c}} / 2\right], \mathbf{0}, S\left[-N_{\mathrm{c}} / 2\right], \ldots, S[-1]\right] \in \mathbb{C}^{N_{\text {dft }}},
$$

is a shifted and zero-padded version of $\mathbf{S}$ in which $\mathbf{0}=$ $[0, \ldots, 0] \in \mathbb{C}^{N_{\mathrm{dft}}-N_{\mathrm{c}}-1}$. According to [17, p. 533], $w(t)$ is typically defined by a Hamming windowing function, thus:

$$
w(t)=\left\{\begin{array}{ll}
\alpha+(1-\alpha) \cos \left(2 \pi t / T_{w}\right) & t \in\left[-T_{w} / 2, T_{w} / 2\right] \\
0 & \text { otherwise }
\end{array},\right.
$$

where $\alpha=0.54$ and $T_{w}=10 T_{s}$ is the adopted window width, which yields to an overall duration of $T_{c}=T+10 T_{s}$ for $s_{\mathrm{c}}(t)$.

Thus, instead of using the widely adopted ideal model in (1), this study adopts the realistic OFDM waveform in (2), hence considering the actual output of the DAC.

\section{B. Sub-carrier power distributions}

The OFDM framework is exploited for creating ToA reference signals having different power spectral densities. This is achieved by considering two sets of power distributions: $\mathcal{U}_{d, N_{\mathrm{c}}}$ and $\mathcal{O}_{N_{\mathrm{a}}, N_{\mathrm{c}}}$. An $\mathcal{U}_{d, N_{\mathrm{c}}}$ distribution defines the sub-carrier content $S[k]$, according to the sub-carrier distance $d$, as:

$$
S[k]=\frac{1}{\sqrt{2 N_{\mathrm{a}}}} \cdot\left\{\begin{array}{ll}
1 & k \in\left\{ \pm\left[N_{\mathrm{c}} / 2-l(d+1)\right], l \in \mathcal{L}_{d, N_{\mathrm{c}}}\right\} \\
0 & \text { otherwise }
\end{array},\right.
$$

where $N_{\mathrm{a}}=\left\lfloor N_{\mathrm{c}} /[2(d+1)]\right\rfloor$ is half the number of nonnull sub-carriers, with $\lfloor\cdot\rfloor$ denoting the floor function, and $\mathcal{L}_{d, N_{\mathrm{c}}}=\left\{0,1, \cdots, N_{\mathrm{a}}-1\right\}$. Each distribution consists of a set of $2 N_{\mathrm{a}}$ active equal power and equal initial phase subcarriers separated by $d$ empty sub-carriers, which are placed symmetrically with respect to the DC starting from the edges of the available bandwidth (i.e. from the sub-carrier index $\left.k=N_{\mathrm{c}} / 2\right)$. Hence, the parameter $d$ controls the density of the active sub-carriers. The value of $d$ is selected from the set $\mathcal{D}_{N_{\mathrm{c}}}=\left\{d \in \mathbb{N}: 0 \leq d<N_{\mathrm{c}} / 2, I_{N_{\mathrm{c}}}(d)=2 d+1\right\}$, where:

$$
I_{N_{\mathrm{c}}}(d)=N_{\mathrm{c}}-2(d+1)\left(\left\lfloor\frac{N_{\mathrm{c}}}{2(d+1)}\right\rfloor-1\right)-1,
$$

is the number of empty sub-carriers determined by the adopted power distribution around the DC sub-carrier. By this choice, increasing the value of $d \in \mathcal{D}_{N_{\mathrm{c}}}$, leads to larger spacing between the used sub-carriers and to a higher energy towards the edge of the band. For each $\mathcal{U}_{d, N_{\mathrm{c}}}$ distribution, a corresponding $\mathcal{O}_{N_{\mathrm{a}}, N_{\mathrm{c}}}$ distribution can be derived by defining $S[k]$ as:

$$
S[k]=\frac{1}{\sqrt{2 N_{\mathrm{a}}}} \cdot \begin{cases}1 & k \in\left\{ \pm l: l \in\left[N_{\mathrm{c}} / 2-N_{\mathrm{a}}+1, N_{\mathrm{c}} / 2\right]\right\} \\ 0 & \text { otherwise }\end{cases}
$$

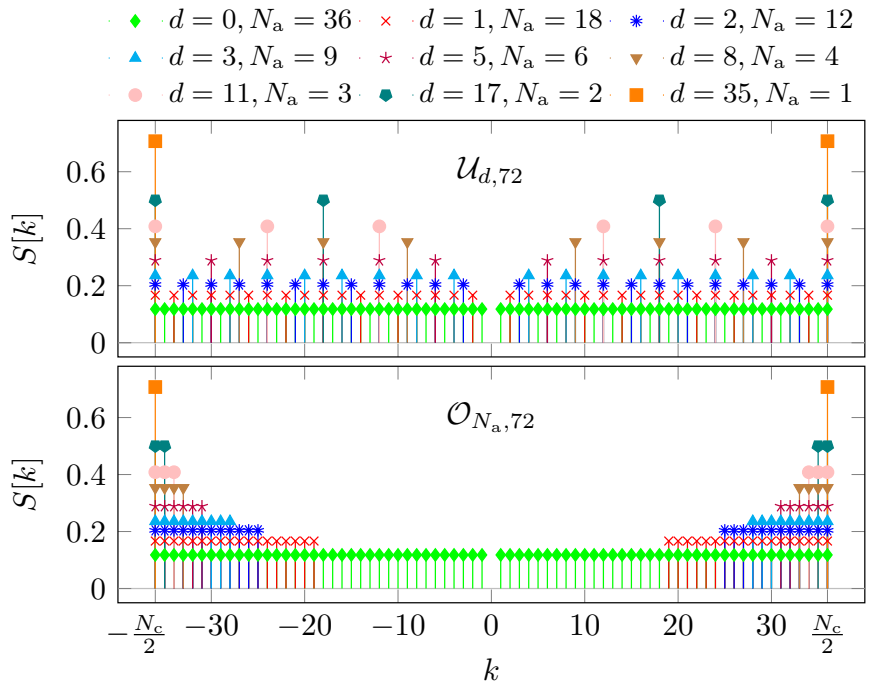

Figure 1. $\mathcal{U}_{d, N_{\mathrm{c}}}$ and $\mathcal{O}_{N_{\mathrm{a}}, N_{\mathrm{c}}}$ power distributions obtained using $N_{\mathrm{c}}=72$ for $d \in \mathcal{D}_{72}=\{0,1,2,3,5,8,11,17,35\}$ and $N_{\mathrm{a}}=\left\lfloor N_{\mathrm{c}} /[2(d+1)]\right\rfloor$.

where, as previously outlined, $N_{\mathrm{a}}$ and $d$ are related. Hence, each $\mathcal{O}_{N_{\mathrm{a}}, N_{\mathrm{c}}}$ distribution consists of $N_{\mathrm{a}}$ sub-carriers contiguously placed both at the negative and positive edges of the available bandwidth. As an example, Fig. 1 shows the $\mathcal{U}_{d, N_{c}}$ and the corresponding $\mathcal{O}_{N_{\mathrm{a}}, N_{\mathrm{c}}}$ distributions for $N_{\mathrm{c}}=72$, which is the number of total available sub-carriers specified in the 3GPP LTE 1.4 MHz channel configuration [18].

\section{TOA ESTIMATION PERFORMANCE EVALUATION}

Consider the estimation of the ToA $\tau$ of the signal $s_{\mathfrak{c}}(t)$ in an AWGN channel, given the received signal:

$$
r(t)=s_{\mathrm{c}}(t-\tau)+z(t), \quad t \in\left[0, T_{\mathrm{o}}\right],
$$

where the observation interval $\left[0, T_{\mathrm{o}}\right]$ is assumed a priori known to the receiver [16], and $z(t)$ is the zero mean complex Gaussian white noise. Defining the signal energy as $\mathcal{E}_{s}=$ $\int_{0}^{T_{c}}\left|s_{\mathrm{c}}(t)\right|^{2} \mathrm{~d} t$ and the noise power spectral density as $\mathcal{N}_{0}$, the signal-to-noise ratio (SNR) at the receiver is $\gamma=\mathcal{E}_{s} / \mathcal{N}_{0}$. If $\hat{\tau}$ is the result of the estimation of $\tau$ given $r(t)$, then the MSEE is given by $\epsilon^{2}=\mathbb{E}\left[(\tau-\hat{\tau})^{2}\right]$. Now, as the main objective of this study, the ToA RMSEE $\epsilon$ obtainable employing the OFDM signal model described in Subsection II-A, is characterized through the sub-carrier power distributions described in Subsection II-B using the widely adopted CRB [15], and the tighter ZZB [16].

\section{A. Bounds on the RMSEE}

If an un-biased estimator is considered, then $\mathbb{E}[\hat{\tau}]=\tau$ and the MSEE is equal to the variance of the estimator $\sigma_{\hat{\tau}}^{2}=\mathbb{E}\left[(\hat{\tau}-\mathbb{E}[\hat{\tau}])^{2}\right]$, which can be lower-bounded using the CRB [15]. Identifying $S_{\mathrm{c}}(f)=\mathcal{F}\left\{s_{\mathrm{c}}(t)\right\}$ as the Fourier transform of $s_{\mathrm{c}}(t)$, the CRB, which can be computed only if $s_{\mathrm{c}}(t)$ satisfies some regularity conditions [15], can be expressed as $\sigma_{\hat{\tau}}^{2} \geq C(\gamma)=1 /\left(2 \gamma \beta^{2}\right)$, where:

$$
\beta^{2}=\frac{1}{\mathcal{E}_{s}} \int_{-\infty}^{\infty}(2 \pi f)^{2}\left|S_{\mathrm{c}}(f)\right|^{2} \mathrm{~d} f,
$$




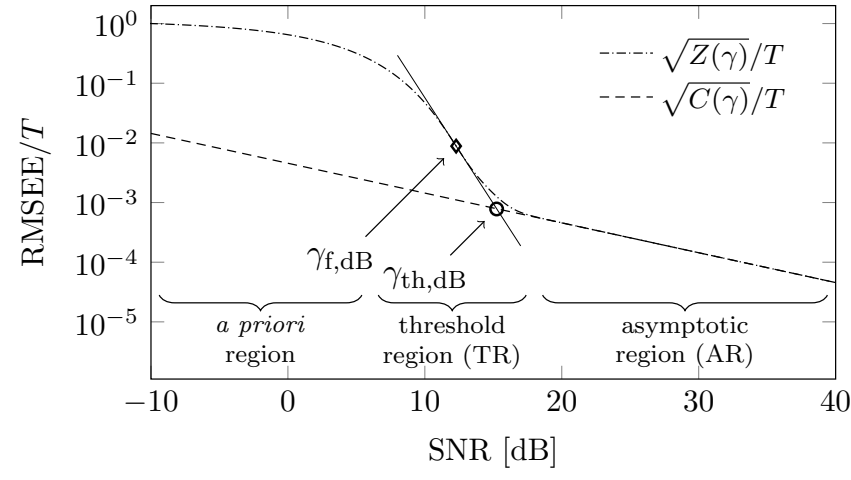

Figure 2. Normalized RMSEE and corresponding bounds for $T_{\mathrm{o}}=4 T$ using the $\mathcal{U}_{2,72}$ power distribution.

is the squared $\mathrm{GB}$, which represents the normalized squared frequency domain power distribution of the signal $s_{\mathrm{c}}(t)$. It can be proved that the CRB is tight only in the AR, i.e. for high values of $\gamma$ [15], [16]. Observe that, intuitively, to increase the GB in (9), one should increase the power concentration at the edges of the available bandwidth. This is the reason for the adoption of the distributions $\mathcal{U}_{d, N_{\mathrm{c}}}$ and $\mathcal{O}_{N_{\mathrm{a}}, N_{\mathrm{c}}}$ defined in Subsection II-B, which allow one to control the GB simultaneously reducing the active sub-carriers' density.

A bound on the MSEE, tighter than the CRB for a wide range of SNR values and holding for all estimators and signals, is the ZZB. The ZZB can be expressed as $\epsilon^{2} \geq Z(\gamma)$, with:

$$
Z(\gamma)=\frac{1}{T_{\mathrm{o}}} \int_{0}^{T_{\mathrm{o}}} \Delta\left(T_{\mathrm{o}}-\Delta\right) Q(\sqrt{\gamma[1-\rho(\Delta)]}) \mathrm{d} \Delta
$$

where $Q(x)=(2 \pi)^{-1 / 2} \int_{x}^{\infty} e^{-u^{2} / 2} \mathrm{~d} u$ and:

$$
\rho(\Delta)=\frac{1}{\mathcal{E}_{s}} \cdot \begin{cases}\int_{0}^{T_{\mathrm{c}}} \mathfrak{R}\left\{s_{\mathrm{c}}^{*}(t-\Delta) s_{\mathrm{c}}(t)\right\} \mathrm{d} t & \Delta \in\left[0, T_{\mathrm{c}}\right] \\ 0 & \text { otherwise }\end{cases}
$$

is the normalized single-sided autocorrelation function of the reference signal $s_{\mathrm{c}}(t)$, with $\mathfrak{R}\{\cdot\}$ and $(\cdot)^{*}$ denoting the real part and the complex conjugate, respectively.

The bounds on the RMSEE obtainable from the CRB and the ZZB are $\sqrt{C(\gamma)}$ and $\sqrt{Z(\gamma)}$, respectively. These bounds, normalized to the ideal signal duration $T$, are shown in Fig. 2 for the $\mathcal{U}_{2,72}$ distribution. The qualitative boundaries between a priori, threshold and asymptotic regions are also highlighted, together with the quantities that are proposed for determining a bound between the threshold and the AR, and that are described in the following section.

\section{B. RMSEE performance figures}

Two quantities are considered for the evaluation of the ToA estimation performance of each power distribution. As one can see from Fig. 2, the asymptotic RMSEE performance is identified by the $\mathrm{CRB}$, which directly depends on the GB $\beta$. Hence, the first considered metric is the normalized GB $\beta T$, which is a dimensionless parameter that quantifies the ToA estimation performance for high SNR values and does not depend on the signal duration.
In addition, for characterizing the signal performance in the TR, a novel performance figure is introduced. Define $\gamma_{\mathrm{dB}}=$ $10 \log _{10} \gamma$, and let $\gamma_{\mathrm{f}, \mathrm{dB}}=10 \log _{10} \gamma_{\mathrm{f}}$ be the maximum value of SNR expressed in $\mathrm{dB}$ for which the logarithmic plot of $\sqrt{Z\left(10^{\gamma_{\mathrm{dB}} / 10}\right)}$ has a convex to concave point of inflection. Defining $\Xi\left(\gamma_{\mathrm{dB}}\right)=Z\left(10^{\gamma_{\mathrm{dB}} / 10}\right)$, the value $\gamma_{\mathrm{f}, \mathrm{dB}}$ can be found by taking the highest solution of:

$$
\frac{\partial^{2}}{\partial \gamma_{\mathrm{dB}}^{2}}\left[\log _{10} \sqrt{\Xi\left(\gamma_{\mathrm{dB}}\right)}\right]=0,
$$

for which the left hand side of (12) changes sign from minus to plus. Using (10), (12) can be rewritten as:

$$
\dot{\Xi}^{2}\left(\gamma_{\mathrm{dB}}\right)=\Xi\left(\gamma_{\mathrm{dB}}\right) \cdot \ddot{\Xi}\left(\gamma_{\mathrm{dB}}\right) \text {, }
$$

where:

$$
\begin{aligned}
\dot{\Xi}\left(\gamma_{\mathrm{dB}}\right)= & \frac{\partial}{\partial \gamma_{\mathrm{dB}}}\left[\Xi\left(\gamma_{\mathrm{dB}}\right)\right]=-\frac{\log 10}{20 \sqrt{2 \pi} T_{\mathrm{o}}} \int_{0}^{T_{\mathrm{o}}}\left\{\Delta\left(T_{\mathrm{o}}-\Delta\right) .\right. \\
& \left.\cdot e^{-\frac{\xi\left(\gamma_{\mathrm{dB}}, \Delta\right)}{2}} \sqrt{\xi\left(\gamma_{\mathrm{dB}}, \Delta\right)}\right\} \mathrm{d} \Delta, \quad(14) \\
\ddot{\Xi}\left(\gamma_{\mathrm{dB}}\right)= & \frac{\partial^{2}}{\partial \gamma_{\mathrm{dB}}^{2}}\left[\Xi\left(\gamma_{\mathrm{dB}}\right)\right]=\frac{\log ^{2} 10}{20^{2} \sqrt{2 \pi} T_{\mathrm{o}}} \int_{0}^{T_{\mathrm{o}}}\left\{\Delta\left(T_{\mathrm{o}}-\Delta\right) .\right. \\
& \left.\cdot e^{-\frac{\xi\left(\gamma_{\mathrm{dB}}, \Delta\right)}{2}} \sqrt{\xi\left(\gamma_{\mathrm{dB}}, \Delta\right)}\left[\xi\left(\gamma_{\mathrm{dB}}, \Delta\right)-1\right]\right\} \mathrm{d} \Delta, \quad(15)
\end{aligned}
$$

with $\xi\left(\gamma_{\mathrm{dB}}, \Delta\right)=10^{\gamma_{\mathrm{dB}} / 10}[1-\rho(\Delta)]$. As depicted in Fig. 2, the novel performance figure is referred to as $\gamma_{\mathrm{th}, \mathrm{dB}}=$ $10 \log _{10} \gamma_{\text {th }}$ and is defined as the intersection between the logarithmic plot of $\sqrt{C\left(10^{\gamma_{\mathrm{dB}} / 10}\right)}$ and the tangent to the logarithmic plot of $\sqrt{Z\left(10^{\gamma_{\mathrm{dB}} / 10}\right)}$ at $\gamma_{\mathrm{f}, \mathrm{dB}}$. In particular, after some algebra, $\gamma_{\mathrm{th}, \mathrm{dB}}$ can be evaluated as:

$\gamma_{\mathrm{th}, \mathrm{dB}}=\frac{\gamma_{\mathrm{f}, \mathrm{dB}} \dot{\Xi}\left(\gamma_{\mathrm{f}, \mathrm{dB}}\right)-\log 10 \cdot \Xi\left(\gamma_{\mathrm{f}, \mathrm{dB}}\right) \cdot \log _{10}\left[2 \Xi\left(\gamma_{\mathrm{f}, \mathrm{dB}}\right) \beta^{2}\right]}{\dot{\Xi}\left(\gamma_{\mathrm{f}, \mathrm{dB}}\right)+\log 10 \cdot \Xi\left(\gamma_{\mathrm{f}, \mathrm{dB}}\right) / 10}$.

This SNR value may be used to reliably approximate the transition point between the TR and AR, where the ZZB becomes tight to the CRB. Hence, signals having a small value of $\gamma_{\text {th }}$ are considered as signals achieving a good RMSEE performance in the TR.

\section{RESULTS}

This section discusses the numerical results which are obtained using $T_{\mathrm{o}}=4 T$ and considering the two cases $\left(N_{\mathrm{c}}, N_{\mathrm{dft}}\right)=(72,128)$ and $\left(N_{\mathrm{c}}, N_{\mathrm{dft}}\right)=(300,512)$, corresponding to the 3GPP LTE specifications for the $1.4 \mathrm{MHz}$ and $5 \mathrm{MHz}$ channel configurations, respectively [18].

Fig. 3 depicts the normalized ZZBs obtained for different $\mathcal{U}_{d, N_{\mathrm{c}}}$ distributions, selecting, for readability purposes, four representative values of $d$ for each value of $N_{\mathrm{c}}$. In Fig. 3, for each plotted curve, the circle marker identifies the SNR threshold $\gamma_{\mathrm{th}, \mathrm{dB}}$ evaluated by (16), while the triangle marker identifies, for comparison purposes, the usual SNR threshold $\delta_{2}$ (reported in $\mathrm{dB}$ at the same ordinate of the corresponding $\gamma_{\mathrm{th}, \mathrm{dB}}$ value), which is evaluated as the solution of the equation $Z\left(\delta_{2}\right)=2 C\left(\delta_{2}\right)$ [19], [20]. Three aspects may be outlined from this figure. Firstly, it is evident that the novel performance figure $\gamma_{\mathrm{th}, \mathrm{dB}}$ effectively evaluates the RMSEE threshold performance of a signal by properly locating the 


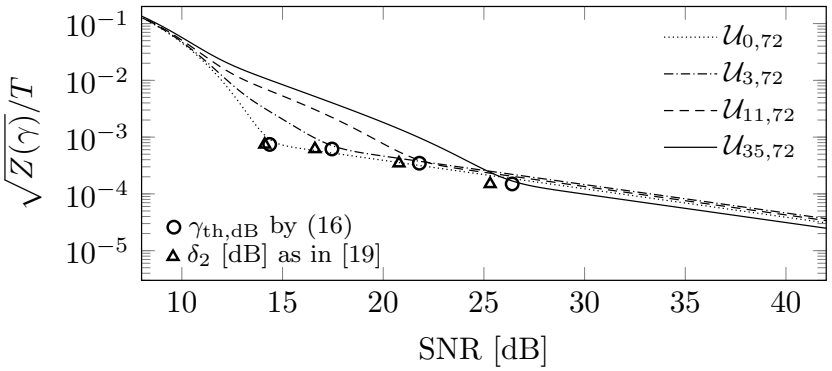

(a) $N_{\mathrm{c}}=72$.

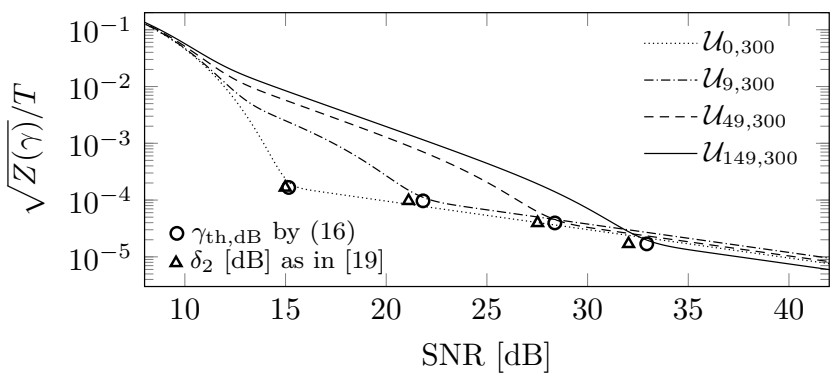

(b) $N_{\mathrm{c}}=300$.

Figure 3. Plots of $\sqrt{Z(\gamma)} / T$ for selected distributions $\mathcal{U}_{d, N_{\mathrm{c}}}$.

boundary between threshold and asymptotic regions. Secondly, $N_{\mathrm{c}}$ strongly influences the asymptotic RMSEE performance, since higher values of $N_{\mathrm{c}}$ lead to an increase of the bandwidth occupied by the sub-carriers with the same inter-channel spacing $\Delta f$. This ultimately determines an increased GB and hence a smaller asymptotic RMSEE. And thirdly, the adopted value of sub-carriers' spacing $d$ strongly influences the RMSEE behavior in the TR. Interestingly, the signal obtaining the best asymptotic performance shows the worst threshold behavior. A direct comparison between $\gamma_{\mathrm{th}, \mathrm{dB}}$ and $\delta_{2}$ shows a satisfactory agreement between the two definitions of the SNR threshold, further revealing that, when the ZZB in the boundary between TR and AR is sufficiently steep, $\gamma_{\mathrm{th}, \mathrm{dB}}$ is able to better capture the point at which the TR itself actually ends. Conversely, further results, not reported for lack of space, reveal that, when the ZZB curve has a flatter transition from the TR to the AR, $\delta_{2}$ better captures the actual end of the TR.

Consider now the normalized GB $\beta T$ and the threshold gain $G_{\mathrm{th}}=\gamma_{\mathrm{th}}^{\max } / \gamma_{\mathrm{th}}$, where $\gamma_{\mathrm{th}}^{\max }$ is the value of $\gamma_{\mathrm{th}}$ when $d=N_{\mathrm{c}} / 2-1$, that is when $N_{\mathrm{a}}=1$. To explore the RMSEE behavior of the different OFDM signals with the spectra determined by the parameters $d$ and $N_{\mathrm{a}}$, Fig. 4 shows how the values of $\beta T$ and $G_{\mathrm{th}}$ vary depending on the values of $N_{\mathrm{a}}$ obtained using $d \in \mathcal{D}_{N_{\mathrm{c}}}$. As one can see, for the distributions $\mathcal{U}_{d, N_{\mathrm{c}}}$ and a fixed $N_{\mathrm{c}}$, the value of $\beta T$ that results from a decrease of the number of active sub-carriers $N_{\text {a }}$ (i.e. an increase of the sub-carriers' spacing $d$ ), is not associated with an improvement in the threshold gain $G_{\mathrm{th}}$, and hence with a reduction of $\gamma_{\mathrm{th}, \mathrm{dB}}$. This reveals a trade-off between $\beta T$ and $G_{\text {th }}$, i.e. between asymptotic and threshold performance, which is even more evident for the distributions $\mathcal{O}_{N_{\mathrm{a}}, N_{\mathrm{c}}}$. This demonstrates that the GB is not the only parameter that should be considered when designing a ToA reference signal [15], since the maximization of the GB does not directly guarantee a

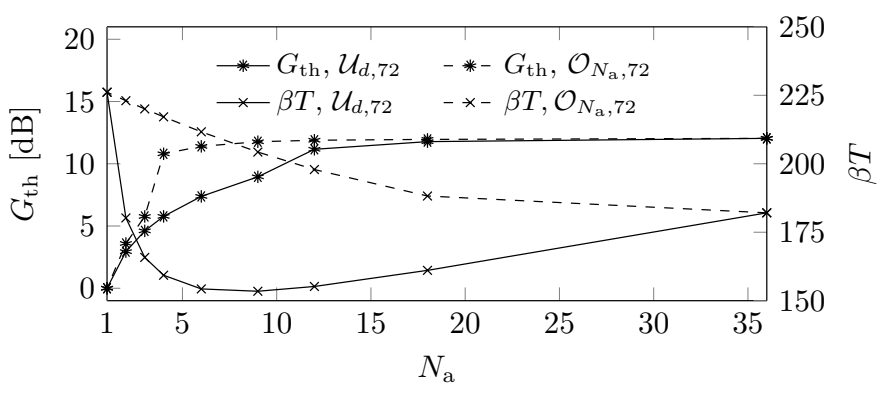

(a) $N_{\mathrm{c}}=72$.

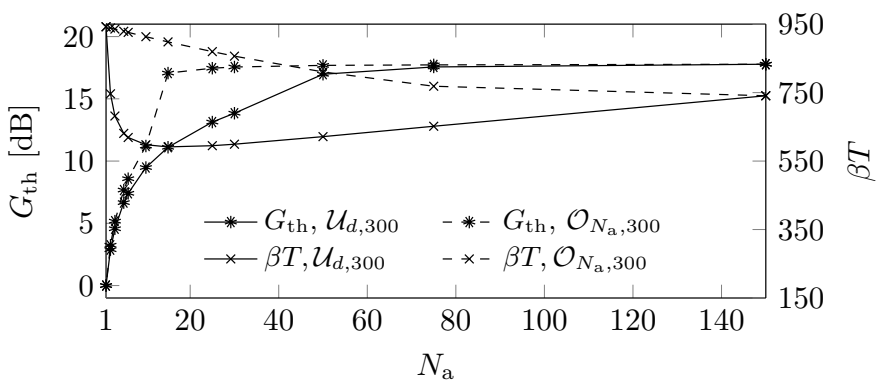

(b) $N_{\mathrm{c}}=300$.

Figure 4. Plots of $G_{\text {th }}(*)$ and $\beta T(\times)$ for the distributions $\mathcal{U}_{d, N_{\mathrm{c}}}$ and $\mathcal{O}_{N_{\mathrm{a}}, N_{\mathrm{c}}}$ with respect to $N_{\mathrm{a}}=\left\lfloor N_{\mathrm{c}} /[2(d+1)]\right\rfloor$, where $d \in \mathcal{D}_{N_{\mathrm{c}}}$.

satisfactory threshold performance. Finally, these results show that an acceptable RMSEE performance in the TR can be achieved using a sufficiently high density of active sub-carriers for the equispaced $\mathcal{U}_{d, N_{\mathrm{c}}}$ distributions, or a sufficiently high number of contiguous active sub-carriers at the edges of the bandwidth for the $\mathcal{O}_{N_{\mathrm{a}}, N_{\mathrm{c}}}$ distributions.

\section{CONCLUSIONS}

The ToA RMSEE performance of realistically modelled OFDM signals has been investigated in the AWGN channel, using a model that considers the waveform directly at the output of the DAC. Two sets of power distributions on the available sub-carriers have been defined to evaluate the RMSEE performance both in the asymptotic and threshold regions by using the CRB and the ZZB. The developed analysis has confirmed that, for a given maximum bandwidth, the power distribution influences the RMSEE both in the asymptotic and threshold regions. Analysis using a novel performance figure, introduced to quantify the RMSEE threshold behavior, has revealed the existence of a trade-off in the performance of the timing measurements of OFDM signals. More precisely, a widely spaced power distribution, concentrated on the edges of the available bandwidth, has the positive effect of determining a small asymptotic RMSEE but the negative effect of giving a poor RMSSE threshold behavior. The analysis has also shown that, to obtain a satisfactory threshold performance with an equispaced sub-carrier power distribution, a high density of active sub-carriers is required. Similarly, a certain number of active sub-carries has to be employed if they have to be placed contiguously on the edges of the available bandwidth. Future work aims to extend the ToA performance analysis to multipath channels, by adopting the presented framework as a reference best-case indication. 


\section{REFERENCES}

[1] Y. G. Li and G. L. Stüber, Orthogonal Frequency Division Multiplexing for Wireless Communications. Springer, 2006.

[2] P. J. Voltz and D. Hernandez, "Maximum likelihood time of arrival estimation for real-time physical location tracking of $802.11 \mathrm{a} / \mathrm{g}$ mobile stations in indoor environments," in Position Location and Navig. Symp., April 2004, pp. 585-591.

[3] O. Bar-Shalom and A. J. Weiss, "Efficient direct position determination of orthogonal frequency division multiplexing signals," IET Radar, Sonar \& Navig., vol. 3, no. 2, pp. 101-111, April 2009.

[4] L. Dai, Z. Wang, J. Wang, and Z. Yang, "Positioning with OFDM signals for the next-generation GNSS," IEEE Trans. Consum. Electron., vol. 56, no. 2, pp. 374-379, May 2010.

[5] R. K. Martin, C. Yan, H. H. Fan, and C. Rondeau, "Algorithms and bounds for distributed TDOA-based positioning using OFDM signals," IEEE Trans. Signal Process., vol. 59, no. 3, pp. 1255-1268, March 2011.

[6] J. A. del Peral-Rosado, J. A. López-Salcedo, G. Seco-Granados, F. Zanier, and M. Crisci, "Preliminary analysis of the positioning capabilities of the positioning reference signal of 3GPP LTE," in Eur. Work. on GNSS Signals and Signal Process., December 2011.

[7] Z. Fei, W. Lu-kai, and F. Xin-yue, "An improving beam-space MUSIC time delay estimation algorithm for OFDM signal," in Int. Conf. on Fuzzy Systems and Knowledge Discovery, May 2012, pp. 2115-2119.

[8] J. A. del Peral-Rosado, J. A. López-Salcedo, G. Seco-Granados, F. Zanier, and M. Crisci, "Achievable localization accuracy of the positioning reference signal of 3GPP LTE," in Int. Conf. on Localization and GNSS, June 2012, pp. 1-6.

[9] Z. He, Y. Ma, and R. Tafazolli, "Improved high resolution TOA estimation for OFDM-WLAN based indoor ranging," IEEE Wireless Commun. Lett., vol. 2, no. 2, pp. 163-166, April 2013.

[10] J. A. del Peral-Rosado, J. A. López-Salcedo, G. Seco-Granados, F. Zanier, and M. Crisci, "Joint maximum likelihood time-delay estimation for lte positioning in multipath channels," EURASIP J. Advances Signal Process., vol. 2014, no. 1, p. 33, 2014.

[11] A. Emmanuele, M. Luise, F. Zanier, and M. Crisci, "Selective accuracy and multiresolution capabilities are intrinsic features of multicarrier waveforms for GNSS," in ESA Work. on Satellite Navig. Technol. \& Europ. Work. on GNSS Signals and Signal Process., Dec 2012, pp. 1-8.

[12] M. D. Larsen, G. Seco-Granados, and A. L. Swindlehurst, "Pilot optimization for time-delay and channel estimation in OFDM systems," in IEEE Int. Conf. on Acoust., Speech, Signal Process., May 2011, pp. 3564-3567.

[13] R. Montalban, J. A. López-Salcedo, G. Seco-Granados, and A. L. Swindlehurst, "Power allocation method based on the channel statistics for combined positioning and communications OFDM systems," in IEEE Int. Conf. on Acoust., Speech, Signal Process., May 2013, pp. 43844388.

[14] A. Shahmansoori, R. Montalban, J. A. López-Salcedo, and G. SecoGranados, "Design of OFDM sequences for joint communications and positioning based on the asymptotic expected CRB," in Int. Conf. on Localization and GNSS, June 2014.

[15] S. M. Kay, Fundamentals of statistical signal processing, volume I: Estimation theory. Prentice Hall, 1993.

[16] D. Chazan, M. Zakai, and J. Ziv, "Improved lower bounds on signal parameter estimation," IEEE Trans. Inf. Theory, vol. 21, no. 1, pp. 9093, 1975.

[17] S. K. Mitra, Digital Signal Processing: A Computer-Based Approach, 3rd ed. McGraw-Hill, 2006.

[18] 3GPP TS 36.211, Evolved Universal Terrestrial Radio Access (EUTRA); Physical channels and modulation (Release 11), 3rd Generation Partnership Project, V11.0.0, 2012.

[19] Z. Xu and B. M. Sadler, "Time delay estimation bounds in convolutive random channels," IEEE J. Sel. Topics Signal Process., vol. 1, no. 3, pp. 418-430, Oct 2007.

[20] B. M. Sadler, N. Liu, and Z. Xu, "Ziv-Zakai bounds on time delay estimation in unknown convolutive random channels," IEEE Trans. Signal Process., vol. 58, no. 5, pp. 2729-2745, May 2010. 